\title{
Avaliação dos preditores de ventilação sob máscara facial difícil (VMF)
}

\author{
Categoria Cirúrgico \\ Centro Universitário São Camilo
}

\author{
Leandro Leal Silva \\ Leonardo Ayres Canga \\ Laura Valquíria Ramos Maita \\ Márcia A. Tedesco
}

Rua Tupiniquins, ํㅜ 51 - Jardim Bandeirantes

Ribeirão Pires - SP - CEP: 09440-010

(11) $96775-6759$

Leandroleal.silva.Ils@gmail.com

Leandro.leal@aluno.saocamilo-sp.br 


\title{
Avaliação dos preditores de ventilação sob máscara facial difícil (VMF)
}

\author{
Categoria Cirúrgico
}

Descritores: ventilação sob máscara facial, preditores da via aérea difícil 


\title{
Avaliação dos preditores de ventilação sob máscara facial difícil (VMF)
}

\author{
SILVA, L.L. ${ }^{1}$; CANGA, L.A. ${ }^{1}$; MAITA, L.V.R. ${ }^{1}$; TEDESCO, M.A. ${ }^{2}$ \\ ${ }^{1}$ Acadêmicos de Medicina do Centro Universitário São Camilo \\ ${ }^{2}$ Especialista em Anestesiologia - Sociedade Brasileira de Anestesiologia
}

\section{RESUMO}

\section{Introdução}

Segundo a ASA, a definição de VMF difícil ocorre quando o anestesiologista não consegue proporcionar uma adequada ventilação com máscara facial por diversos fatores e o profissional precisa previamente estar preparado para lidar com essa situação. Portanto, este trabalho tem como objetivo avaliar os preditores de VMF difícil e a possível incidência a partir de uma amostra populacional.

\section{Métodos}

Estudo observacional, prospectivo, realizado em Hospitais do ABC Paulista. Critérios de inclusão foram pacientes que seriam submetidos a cirurgias eletivas sob anestesia geral com idade entre 18 a 65 anos, de ambos os sexos, IMC entre 18-40, ASA P1 e P2, os de exclusão foram cirurgias de emergência, gestantes, cirurgias envolvendo vias aéreas, obesos mórbidos, ASA P3-P6 e antecedente pessoal de via aérea difícil.

Os dados foram dispostos em planilha e avaliados os preditores de VMF difícil através de análise estatística com o SPSS 17.0 for Windows. Fora aplicado o teste t de Student, teste de qui-quadrado e teste exato de Fisher, quando o caso.

\section{Resultados}

79 pacientes avaliados. Média de 40,51 anos, com desvio-padrão de 11,91 anos. 64,6\% pacientes do sexo feminino. A análise de acordo com a escala de Han evidenciou que $62,3 \%$ recebeu a VMF sem dificuldades, enquanto que $18,2 \%$ apresentou ventilação inadequada e/ou requereu dois profissionais para o fazer.

\section{Conclusão}

Diversos preditores da VMF difícil são descritos na literatura. Observa-se neste estudo que pacientes obesos, com barba, índices maiores de Mallampati e com circunferência cervical maior que $40 \mathrm{~cm}$ apresentam risco maior de apresentarem dificuldade na VMF.

Palavras-chave: ventilação sob máscara facial, preditores da via aérea difícil 


\section{Avaliação dos preditores de ventilação sob máscara facial difícil}

\section{INTRODUÇÃO}

A ventilação sob máscara facial (VMF) é uma das habilidades básicas dos anestesiologistas. [1] Muitas vezes é o ponto inicial para as anestesias gerais e tem grande importância para manter a oxigenação em procedimentos de intubação difícil. [1]

Segundo a Sociedade Americana de Anestesiologia (American Society of Anesthesiologists - ASA) a definição de VMF difícil ocorre quando o anestesiologista não consegue proporcionar uma adequada ventilação por máscara com inadequada vedação, escape de gás excessivo, ou grande resistência para entrada ou saída do gás, podendo ser problema único ou associados entre eles. [1] Há outros parâmetros avaliados, todavia, são operadores dependentes, sendo influenciada também pelo nível de experiência do profissional. [1]

Desse modo, a fim de estabelecer um parâmetro para facilitar uma correta comunicação entre profissionais, Han et. al. representou em uma escala graduada de 1 a 4 os estágios da dificuldade da VMF (Quadro 1). [2]

Quadro 1: Escala de classificação e descrição de ventilação com máscara facial

\begin{tabular}{|c|l|}
\hline Classificação & \multicolumn{1}{|c|}{ Descrição } \\
\hline Grau 1 & ventilação sem dificuldades \\
\hline Grau 2 & ventilação com cânula oral associada ou não a relaxante muscular \\
\hline Grau 3 & $\begin{array}{l}\text { ventilação inadequada para manter a oxigenação; instável; requer } \\
\text { duas pessoas }\end{array}$ \\
\hline Grau 4 & $\begin{array}{l}\text { ventilação impossível, notada pela ausência de CO2 expirado e } \\
\text { pela falta de movimentos perceptíveis da caixa torácica, mesmo } \\
\text { com o emprego de VPP; apesar de auxiliares. }\end{array}$ \\
\hline
\end{tabular}

VPP: Ventilação com pressão positiva

Além disso, é importante para o profissional estar preparado para lidar com uma VMF difícil. [1] Há diversos fatores que podem ser avaliados no paciente. [1] Os principais preditores de VMF difícil podem ser representados pelo mnemônico MMMMASK (Tabela 1). [1]

Tabela 1: Mnemônico com os principais preditores de VMF

\begin{tabular}{l|l}
\hline \multicolumn{2}{c}{ MMMMASK } \\
\hline $\mathbf{M}$ & Masculino \\
\hline $\mathbf{M}$ & Máscara com vedação ruim por barba ou desdentado \\
\hline $\mathbf{M}$ & Mallampati grau 3 ou 4 \\
\hline $\mathbf{M}$ & Mandíbula protrusa \\
\hline $\mathbf{A}$ & Ancião (Age, idade) \\
\hline $\mathbf{S}$ & Sons de apneia obstrutiva ou ronco \\
\hline $\mathbf{K}$ & Kilogramas (peso) \\
\hline
\end{tabular}

Dessa forma, em vista da importância da VMF e o número limitado de obras sobre este tema, o presente trabalho tem como objetivo avaliar os preditores de VMF difícil e a possível incidência a partir de uma amostra populacional. 


\section{METODOLOGIA}

Estudo observacional, prospectivo, realizado a partir da confirmação de participação na pesquisa por parte de pacientes de Hospitais da Região do ABC Paulista que concordaram em assinar o Termo de Consentimento Livre e Esclarecido (TCLE).

O estudo foi aprovado pelo comitê de ética em pesquisa.

Para que o indivíduo pudesse participar da pesquisa precisaram informar alguns dados a fim de que os preditores de via aérea difícil pudessem ser avaliados:

- Cirurgia a ser submetido;

- Peso em Kg;

- IMC;

- Idade (em anos);

- Sexo;

- Comorbidades;

- Classificação ASA;

- História de via aérea difícil;

- Presença de barba;

- Ausência de dentes;

- Ronco ou apneia do sono;

- Limitação da protrusão mandibular;

- Dentição profusa;

- Teste de mordida do lábio superior - ULBT, do inglês Upper lip bite test;

- Índice de Mallampati Modificado e

- Mobilidade cervical.

Os critérios de inclusão foram pacientes que seriam submetidos a cirurgias eletivas sob anestesia geral com idade entre 18 a 65 anos, de ambos os sexos, com IMC entre 1840, ASA P1 e P2.

Os critérios de exclusão foram cirurgias de emergência, gestantes, cirurgias envolvendo vias aéreas, obesos mórbidos, ASA P3-P6 e antecedente pessoal de via aérea difícil.

Após a anamnese e exame físico foi observado a indução anestésica de todos os participantes (que ocorreu de forma padronizada) durante a tentativa de ventilação sob máscara facial.

A padronização da técnica anestésica do presente estudo aos participantes consistiu em:

1) Não administração de medicação pré-anestésica;

2) Monitorização da oximetria de pulso, cardioscópio e pressão arterial não invasiva;

3) Posicionamento adequado com coxins;

4) Pré-oxigenação adequada seguida de indução anestésica com administração de Fentanil 3-5mcg/kg, Propofol $2 \mathrm{mg} / \mathrm{kg}$ e Atracúrio 0,5mg/kg;

5) Ventilação (após indução anestésica) sob máscara facial de tamanho adequado, sendo avaliados quanto a qualidade da ventilação segundo a Classificação de Han

Com a coleta, os dados foram dispostos em uma planilha do Microsoft Office Excel 365 e foram avaliados os preditores de VMF difícil através de análise estatística com 0 
software SPSS 17.0 for Windows. Os dados foram analisados em frequência simples, cálculo das médias, desvios-padrão e mediana. As análises estatísticas foram analisadas utilizando o teste t de Student, teste de qui-quadrado e teste exato de Fisher, quando o caso. O nível de significância utilizados para os testes foi de $5 \%$.

\section{RESULTADOS}

Foram avaliados 79 pacientes com idade entre 18 e 65 anos (média de 40,51 anos com desvio-padrão de 11,91 anos e mediana de 39,00 anos) (Tabela 2).

Cinquenta e um (64,6\%) pacientes eram do sexo feminino (Tabela 3).

Tabela 2. Principais variáveis dos participantes da pesquisa

\begin{tabular}{|c|c|c|c|c|c|c|}
\hline Variável & $\mathbf{n}$ & Média & $d p$ & Mediana & Mínimo & Máximo \\
\hline Idade & 79 & 40,51 & 11,91 & 39,00 & 18,00 & 65,00 \\
\hline Peso & 79 & 78,30 & 16,54 & 75,00 & 39,00 & 120,00 \\
\hline Altura & 79 & 1,67 & 0,11 & 1,65 & 1,49 & 1,95 \\
\hline IMC & 79 & 27,91 & 4,99 & 27,00 & 17,00 & 41,00 \\
\hline
\end{tabular}

Tabela 3: Frequências absolutas e relativas do sexo e comorbidades dos participantes

\begin{tabular}{|c|c|c|c|}
\hline Variável & Classificação & $\mathrm{n}$ & $\%$ \\
\hline \multirow[t]{2}{*}{ Sexo } & Feminino & 51 & $\overline{64,6}$ \\
\hline & Masculino & 28 & 35,4 \\
\hline \multirow[t]{13}{*}{ Comorbidades } & HAS & 14 & 17,7 \\
\hline & Hipotiroidismo & 4 & 5,1 \\
\hline & Não Tabagista & 69 & 87,3 \\
\hline & Ex tabagista & 1 & 1,3 \\
\hline & Tabagista & 9 & 11,4 \\
\hline & Obesidade & 5 & 6,3 \\
\hline & DM & 3 & 3,8 \\
\hline & Ansiedade & 3 & 3,8 \\
\hline & Gastrite & 3 & 3,8 \\
\hline & Depressão & 2 & 2,5 \\
\hline & Asma & 2 & 2,5 \\
\hline & Etilismo & 2 & 2,5 \\
\hline & Outros & 6 & 7,6 \\
\hline
\end{tabular}


A Tabela 4 apresenta a análise das variáveis clínicas dos pacientes participantes da pesquisa.

Tabela 4: Frequências absolutas e relativas das variáveis clínicas dos participantes.

\begin{tabular}{|c|c|c|c|}
\hline Variável & Classificação & $\mathrm{n}$ & $\%$ \\
\hline \multirow[t]{2}{*}{$\overline{A S A}$} & 1 & 38 & 48,1 \\
\hline & 2 & 41 & 51,9 \\
\hline Ap de VAD & & 1 & 1,3 \\
\hline Barba & & 14 & 17,7 \\
\hline Ausência de dentes & & 37 & 46,8 \\
\hline Apneia & & 32 & 40,5 \\
\hline $\begin{array}{l}\text { Limitação da protusão } \\
\text { mandibular }\end{array}$ & & 13 & 16,5 \\
\hline \multirow[t]{3}{*}{ Dentição protusa } & Normal & 66 & 83,5 \\
\hline & Moderada & 11 & 13,9 \\
\hline & Severa & 2 & 2,5 \\
\hline \multirow[t]{3}{*}{ ULBT } & 1 & 66 & 83,5 \\
\hline & II & 11 & 13,9 \\
\hline & III & 2 & 2,5 \\
\hline \multirow[t]{4}{*}{ Mallampati } & 1 & 38 & 48,0 \\
\hline & 2 & 30 & 38,0 \\
\hline & 3 & 10 & 12,7 \\
\hline & 4 & 1 & 1,3 \\
\hline \multirow[t]{2}{*}{ Mobilidade cervical } & Normal & 75 & 96,2 \\
\hline & Limitada & 3 & 3,8 \\
\hline
\end{tabular}

Segundo a análise dos participantes de acordo com a escala de Han pode-se observar que $62,3 \%$ recebeu a VMF sem dificuldades, enquanto que $18,2 \%$ apresentou ventilação inadequada e/ou requereu dois profissionais para o fazer (Tabela 5).

Tabela 5: Classificação dos participantes segundo escala de Han

\begin{tabular}{lll}
\hline Classificação de Han & $\mathrm{n}$ & $\%$ \\
\hline 1 & 48 & 62,3 \\
2 & 15 & 19,5 \\
3 & 14 & 18,2
\end{tabular}




\section{DISCUSSÃO}

A partir dos resultados foram analisados os preditores que foram mais assertivos a detectar as possíveis dificuldades no manejo da VMF através da análise estatística (Tabela 6) segundo a classificação de Han.

Tabela 6: Cirurgia, sexo e comorbidades, segundo o grupo Han.

\begin{tabular}{|c|c|c|c|c|c|c|c|c|}
\hline \multirow[b]{3}{*}{ Variável } & & \multicolumn{6}{|c|}{ Han } & \multirow[b]{3}{*}{$p$} \\
\hline & & $1(n$ & & $2(n$ & 15) & $3(n$ & $=14)$ & \\
\hline & Classificação & $\mathrm{n}$ & $\%$ & $\mathrm{n}$ & $\%$ & $\mathrm{n}$ & $\%$ & \\
\hline \multirow[t]{3}{*}{ Cirurgia } & & & & & & & & $0,523^{(2)}$ \\
\hline & CCC VDL & 35 & 72,9 & 11 & 73,3 & 8 & 57,1 & \\
\hline & Outras & 13 & 27,1 & 4 & 26,7 & 6 & 42,9 & \\
\hline \multirow[t]{3}{*}{ Sexo } & & & & & & & & $0,001^{(1)}$ \\
\hline & Feminino & 36 & $75,0^{* *}$ & 10 & $66,7^{* *}$ & 3 & 21,4 & \\
\hline & Masculino & 12 & 25,0 & 5 & 33,3 & 11 & 78,6 & \\
\hline \multicolumn{9}{|c|}{ Comorbidades } \\
\hline & HAS & 6 & 12,5 & 3 & 20,0 & 5 & 35,7 & $0,141^{(2)}$ \\
\hline & Hipotiroidismo & 2 & 4,2 & 2 & 13,3 & 0 & 0,0 & $0,311^{(2)}$ \\
\hline & Tabagismo Não & 43 & 89,6 & 11 & 73,3 & 13 & 92,9 & $0,313^{(2)}$ \\
\hline & Ex & 1 & 2,1 & 0 & 0,0 & 0 & 0,0 & \\
\hline & Sim & 4 & 8,3 & 4 & 26,7 & 1 & 7,1 & \\
\hline & Obesidade & 1 & 2,1 & 2 & 13,3 & 2 & 14,3 & $0,085^{(2)}$ \\
\hline & DM & 1 & 2,1 & 1 & 6,7 & 1 & 7,1 & $0,316^{(2)}$ \\
\hline & Ansiedade & 2 & 4,2 & 1 & 6,7 & 0 & 0,0 & $0,764^{(2)}$ \\
\hline & Gastrite & 2 & 4,2 & 1 & 6,7 & 0 & 0,0 & $0,764^{(2)}$ \\
\hline & Depressão & 1 & 2,1 & 1 & 6,7 & 0 & 0,0 & $0,615^{(2)}$ \\
\hline & Asma & 0 & 0,0 & 1 & 6,7 & 1 & 7,1 & $0,139^{(2)}$ \\
\hline & Etilismo & 1 & 2,1 & 1 & 6,7 & 0 & 0,0 & $0,615^{(2)}$ \\
\hline & Outros & 5 & 10,4 & 0 & 0,0 & 1 & 7,1 & $0.612^{(2)}$ \\
\hline
\end{tabular}

De acordo com a tabela 6 é possível observar que há diferença significativa entre os grupos em relação a variável sexo, visto que o grupo 3 apresenta menor porcentagem de casos de ventilação inadequada para manter a oxigenação sendo em indivíduos do sexo feminino quando comparado aos grupos 1 e 2, com o teste do qui-quadrado de 0,001 , enquanto que os grupos 1 e 2 não apresentam diferença significativa entre si ( $p$ insignificante: $p=0,558)$. 
Esse resultado é similar quando comparado com a literatura. Conforme o estudo de Kheterpal e col. APUD Martins et. al. [2] identificaram 5 (cinco) preditores que influenciavam diretamente a VMF difícil sendo eles (na ordem decrescente de importância):

- Alterações do pescoço por radiação;

- Sexo masculino;

- SAOS;

- Mallampati III/IV;

- Presença de barba.

Além dos preditores citados acima, outros também são importantes para influenciar a MVF, conforme pode ser observado na Tabela 7.

Tabela 7: Valores descritivos das variáveis demográficas, segundo o grupo de Han.

\begin{tabular}{lllll}
\hline & \multicolumn{3}{c}{ Han } & \multirow{2}{*}{ Variável } \\
\cline { 2 - 4 } & $1(\mathrm{n}=48)$ & $2(\mathrm{n}=15)$ & $3(\mathrm{n}=14)$ & $\mathrm{p}^{*}$ \\
\hline Idade & $38,02 \pm 11,28$ & $44,67 \pm 10,40$ & $46,36 \pm 12,93$ & 0,024 \\
Peso & $73,17 \pm 14,49$ & $86,53 \pm 16,99$ & $89,04 \pm 15,45$ & $<0,001$ \\
Altura & $1,65 \pm 0,10$ & $1,69 \pm 0,12$ & $1,73 \pm 0,10$ & 0,037 \\
IMC & $26,75 \pm 4,03$ & $30,50 \pm 5,95$ & $29,67 \pm 5,84$ & 0,014 \\
\hline
\end{tabular}

(*) Nível descritivo de probabilidade da Análise de Variância a um fator

Portanto, através da análise da tabela acima pode-se afirmar que os grupos diferem em relação a idade, peso, altura e IMC $(p<0,05)$.

Diversos estudos indicam que IMC a partir de $26 \mathrm{~kg} / \mathrm{m} 2$ são predisponentes de VMF difícil, como também a própria obesidade [1] enquanto que há estudos que afirmam que IMC maior que $30 \mathrm{~kg} / \mathrm{m} 2$ é predisponente para grau 3 na escala de classificação de Han. [2].

No estudo de Kheterpal e col. também foi observado a incidência de indivíduos classificados segundo os graus de Han: grau $1=77,4 \%$ dos casos; grau $2=21,1 \%$; grau $3=1,4 \%$ e grau $4=0,16 \%$, sendo que o número total de participantes foi de 22660 indivíduos. [2] Neste estudo, apesar do menor n, também houve prevalência do grau 1 na classificação dos indivíduos, conforme observado na tabela 5.

A Tabela 8 apresenta as variáveis clínicas segundo a classificação de Han. Pode-se analisar que os grupos 1, 2 e 3 diferem em relação ao ASA (segundo o teste de Fisher), Barba, Mallampati e Mobilidade cervical (segundo o teste do Qui-quadrado).

Estudos indicam que a presença de barba (único fator modificável), Mallampati III e IV, como também observado nesse estudo, são preditores independentes para grau 3 de Han. [2] Alguns autores recomendam que peça ao paciente fazer o movimento de morder o lábio superior para verificar a protusão de mandíbula. [2]

Foi compatível com a literatura o resultado aqui observado em relação à ausência de dentes: apesar de poder contribuir para uma VMF difícil por escape de gás, não é possível afirmar que a ausência de dentes é um previsor independente. 
Tabela 8: Frequências das variáveis clínicas, segundo o grupo de Han.

\begin{tabular}{|c|c|c|c|c|c|c|c|c|}
\hline \multirow[b]{3}{*}{ Variável } & \multirow[b]{3}{*}{ Classificação } & \multicolumn{6}{|c|}{ Han } & \multirow[b]{3}{*}{$p$} \\
\hline & & \multicolumn{2}{|c|}{$1(n=48)$} & \multicolumn{2}{|c|}{$2(n=15)$} & \multicolumn{2}{|c|}{$3(n=14)$} & \\
\hline & & $\bar{n}$ & $\%$ & $\mathrm{n}$ & $\%$ & $\mathrm{n}$ & $\%$ & \\
\hline \multirow[t]{3}{*}{ ASA } & & & & & & & & $0,004^{(1)}$ \\
\hline & 1 & 29 & 60,4 & 2 & $13,3^{* *}$ & 5 & $35,7^{* *}$ & \\
\hline & 2 & 19 & 39,6 & 13 & 86,7 & 9 & 64,3 & \\
\hline $\begin{array}{l}\text { Ap de } \\
\text { VAD }\end{array}$ & & 1 & 2,1 & 0 & 0,0 & 0 & 0,0 & $1,000^{(2)}$ \\
\hline Barba & & 4 & 8,3 & 3 & 20,0 & 7 & 50,0 & $0,002^{(2)}$ \\
\hline $\begin{array}{l}\text { Ausência } \\
\text { de dentes }\end{array}$ & & 19 & 39,6 & 8 & 53,3 & 10 & 71,4 & $0,100^{(1)}$ \\
\hline Apneia & & 18 & 37,5 & 5 & 33,3 & 9 & 64,3 & $0,156^{(1)}$ \\
\hline $\begin{array}{l}\text { Limitação d } \\
\text { mandibular }\end{array}$ & a protusão & 8 & 16,7 & 2 & 13,3 & 3 & 21,4 & $0,828^{(2)}$ \\
\hline \multirow{4}{*}{$\begin{array}{l}\text { Dentição } \\
\text { protrusa }\end{array}$} & & & & & & & & $0,367^{(2)}$ \\
\hline & Normal & 41 & 85,4 & 12 & 80,0 & 11 & 78,6 & \\
\hline & Moderada & 7 & 14,6 & 2 & 13,3 & 2 & 14,3 & \\
\hline & Severa & 0 & 0,0 & 1 & 6,7 & 1 & 7,1 & \\
\hline
\end{tabular}

$\begin{array}{lllllllll}\text { ULBT } & & & & & & & & 0,075^{(2)} \\ & \text { I II } & 42 & 87,5 & 13 & 86,6 & 9 & 64,3 & \\ \text { Mallampati } & 6 & 12,5 & 1 & 6,7 & 4 & 28,6 & \\ & & 0 & 0,0 & 1 & 6,7 & 1 & 7,1 & \\ \text { III } & 29 & 60,4 & 4 & 26,7 & 3 & 21,4 & \\ \text { 2 } & 15 & 31,3 & 9 & 60,0 & 6 & 42,9 & \\ 3 & 4 & 8,3 & 2 & 13,3 & 4 & 28,6 & \\ 4 & 0 & 0,0 & 0 & 0,0 & 1 & 7,1 & \end{array}$

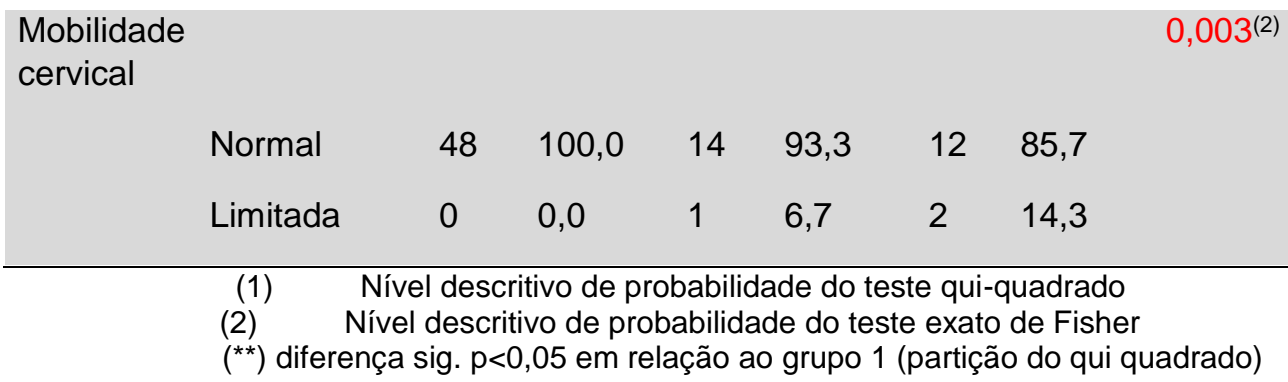




\section{CONCLUSÃO}

A VMF é uma prática recorrente na anestesiologia e a identificação precoce de parâmetros que indiquem a VMF difícil é fundamental para diminuir taxas de morbimortalidade.

Diversos preditores de tal condição são descritos na literatura, e pode-se observar neste estudo que pacientes obesos, com barba e com índices maiores de Mallampati apresentam risco maior de apresentarem dificuldade na VMF.

\section{REFERÊNCIAS}

[1] HOLLAND, Dr Jonathan; DONALDSON, Dr Will. DIFFICULT MASK VENTILATION. 2015. Edited by Dr Luke Baitch and Dr Maytinee Lilaonitkul - The World Federation of Societies of Anaesthesiologists. Disponível em: <https://www.wfsahq.org/components/com_virtual_library/media/739b4e5bd2985bd569 7336592ad5e815-8d652c871b5d27c03f72e56bc43de848-321-Difficult-maskventilation.pdf>. Acesso em: 05 jan. 2019.

[2] ORTENZI, Antonio Vanderlei. Como reconhecer uma via aérea difícil. In: MARTINS, Márcio de Pinho; MORAES, José Mariano Soares de; PIRES, Oscar César. CONTROLE DA VIA AÉREA. Rio de Janeiro: Sociedade Brasileira de Anestesiologia/sba, 2012. Cap. 2. p. 21-29. Disponível em: <http://carraretto.med.br/wpcontent/uploads/2016/06/Via-Aerea_CVA2012.pdf>. Acesso em: 05 jan. 2019. 